\title{
S-2/RES/1. THE GRAVE SITUATION \\ OF HUMAN RIGHTS IN LEBANON CAUSED \\ BY ISRAELI MILITARY OPERATIONS
}

[Con motivo del conflicto desencadenado por la captura de soldados israelíes por miembros de la guerrilla Hezbollah el 12 de julio del 2006, las fuerzas israelíes invadieron y bombardearon Líbano, con un penoso saldo de pérdidas de cientos de vidas humanas de civiles y de militares, además de cuantiosos daños materiales por los bombardeos en Líbano e Israel. El conflicto, secuela al parecer interminable de un conflicto señero en Medio Oriente, puso nuevamente al mundo al borde de una conflagración. Los consejos de seguridad y de derechos humanos de la Organización de Naciones Unidas adoptaron sendas resoluciones.

En el caso de la Resolución A/HRC/S-2/L.1, adoptada por el Consejo de Derechos Humanos, México participó en la gestión y emitió su voto, así como la explicación del sentido que le dio, que aquí reproducimos. Dicha resolución fue adoptada por 27 votos a favor (Algeria, Arabia Saudita, Argentina, Azerbaijan, Bahrein, Bangladesh, Brasil, China, Cuba, Ecuador, Federación Rusa, India, Indonesia, Jordania, Malasia, Mali, Marruecos, Mauricio, México, Paquistán, Perú, Senegal, Sri Lanka, Sudáfrica, Túnez, Uruguay y Zambia) como se puede ver, con el voto de México; 11 en contra (Alemania, Canadá, Finlandia, Francia, Japón, Holanda, Irlanda del Norte, Polonia, Reino Unido de la Gran Bretaña, República Checa, Rumania y Ucrania) y ocho abstenciones (Camerún, Gabón, Ghana, Guatemala, Nigeria, Filipinas, República de Corea, Suiza).

En esta ocasión, el voto mexicano fue claro y contundente a favor de la resolución, no obstante su posición dubitativa en el momento de explicar la razón de su voto ("hubiera sido deseable que la resolución también incluyera una condena a los actos de violencia cometidos por las milicias de Hezbollah y a la necesidad de detener los ataques a la población civil israelí").*]

[* Manuel Becerra Ramírez.]

Anuario Mexicano de Derecho Internacional, vol. VII, 2007, pp. $857-866$ 


\section{The Human Rights Council,}

Reaffirming the purposes and principles contained in the Charter of the United Nations,

Reaffirming also the Universal Declaration of Human Rights and the Vienna Declaration and Programme of Action, and recalling the International Covenant on Civil and Political Rights, the International Covenant on Economic, Social and Cultural Rights, the Convention on the Rights of the Child and other human rights instruments,

Acknowledging that peace and security, development and human rights are the pillars of the United Nations system,

Recalling General Assembly resolution 60/251 of 15 March 2006 in which the Assembly decided that the Human Rights Council:

(a) Should address situations of violations of human rights, including gross and systematic violations, and make recommendations thereon; and

(b) Shall respond promptly to human rights emergencies,

Guided by the Charter of the United Nations, relevant human rights instruments and international humanitarian law, in particular the Hague Conventions of 1899 and 1907 on the Laws and Customs of War on Land which prohibit attacks and bombardment of civilian populations and objects and lay down obligations for general protection against dangers arising from military operations against civilian objects, hospitals, relief materials and means of transportation

Recalling the commitments of the High Contracting Parties to the Geneva Conventions of 12 August 1949 and the Additional Protocols thereto,

Reaffirming that each High Contracting Party to the Geneva Convention relative to the Protection of Civilian Persons in Time of War (Fourth Geneva Convention) is under obligation to take action against persons alleged to have committed or to have ordered the commission of grave breaches of the Convention, and recalling the Convention on the Non-Applicability of Statutory Limitations to War Crimes and Crimes against Humanity,

Emphasizing that human rights law and international humanitarian law are complementary and mutually reinforcing,

Stressing that the right to life constitutes the most fundamental of all human rights,

Condemning Israeli military operations in Lebanon, which constitute gross and systematic human rights violations of the Lebanese people,

Appalled at the massive violations of the human rights of the people of Lebanon by Israel resulting in the massacre of thousands of civilians, injuries, extensive damage to civilian infrastructure, displacement of 1 million people, and 
outflows of refugees fleeing heavy shelling and bombardment against the civilian population,

Strongly condemning the indiscriminate and massive Israeli air strikes, in particular on the village of Qana on 30 July 2006, and the targeting of United Nations peacekeepers at the United Nations observer post in southern Lebanon on 25 July 2006 ,

Taking note of the strong condemnation by the United Nations High Commissioner for Human Rights of the killing of civilians in Qana, her call to take measures to protect civilian lives and civilian objects and her reiteration of the need for independent investigation, with the involvement of international experts,

Noting the extreme concern expressed by the Representative of the Secretary-General on human rights of internally displaced persons, the Special Rapporteur on extrajudicial, summary or arbitrary executions, the Special Rapporteur on the right of everyone to the enjoyment of the highest attainable standard of physical and mental health, the Special Rapporteur on adequate housing as a component of the right to an adequate standard of living, and the Special Rapporteur on the right to food about the continuing adverse impact on the human rights and the humanitarian situation of the civilian population in Lebanon,

Emphasizing that attacks and killings of innocent civilians and the destruction of houses, property and infrastructure in Lebanon are a breach of the principles of the Charter of the United Nations, international law and international humanitarian law as well as are flagrant violations of human rights,

Recognizing the urgent need to address the dire humanitarian situation in Lebanon, including through the immediate lifting of the blockade of Lebanon imposed by Israel,

Noting with concern the environmental degradation caused by Israeli strikes against power plants and their adverse impact on health,

Concerned at the targeting of the communication and media networks in Lebanon,

Outraged at the continuing senseless killings by Israel, with impunity, of children, women, the elderly and other civilians in Lebanon,

1. Strongly condemns the grave Israeli violations of human rights and breaches of international humanitarian law in Lebanon;

2. Condemns the massive bombardment of Lebanese civilian populations, especially the massacres in Qana, Marwaheen, Al Duweir, Al Bayadah, Al Qaa, Chiyah, Ghazieh and other towns of Lebanon, causing thousands of deaths and injuries, mostly among children and women, and the displacement of 1 million 
civilians, according to a preliminary assessment, thus exacerbating the magnitude of the human suffering of the Lebanese;

3. Also condemns the Israeli bombardment of vital civilian infrastructure resulting in extensive destruction and heavy damage to public and private properties;

4. Calls upon Israel to abide, immediately and scrupulously, by its obligations under human rights law, in particular the Convention on the Rights of the Child, and international humanitarian law;

5. Urges all concerned parties to respect the rules of international humanitarian law, to refrain from violence against the civilian population and to treat under all circumstances all detained combatants and civilians in accordance with the Geneva Conventions;

6. Also calls upon Israel to immediately stop military operations against the civilian population and civilian objects resulting in death and destruction and serious violations of human rights;

7. Decides to urgently establish and immediately dispatch a high-level commission of inquiry comprising of eminent experts on human rights law and international humanitarian law, including the possibility of inviting the relevant United Nations special procedures to be nominated to the Commission:

(a) To investigate the systematic targeting and killings of civilians by Israel in Lebanon;

(b) To examine the types of weapons used by Israel and their conformity with international law;

(c) To assess the extent and deadly impact of Israeli attacks on human life, property, critical infrastructure and the environment;

8. Requests the Secretary-General and the United Nations High Commissioner for Human Rights to provide all administrative, technical and logistical assistance required to enable the Commission of Inquiry to fulfil its mandate promptly and efficiently;

9. Calls upon the international community urgently to provide the Government of Lebanon with humanitarian and financial assistance to enable it to deal with the worsening humanitarian disaster, rehabilitation of victims, return of displaced persons and restoration of the essential infrastructure;

10. Requests the Commission of Inquiry to report to the Council no later than 1 September 2006 on progress made towards the fulfilment of its mandate. 


\section{EXPLICACIÓN DEL VOTO DE MÉXICO. SEGUNDA SESIÓN ESPECIAL DEL CONSEJO DE DERECHOS HUMANOS. "LA GRAVE SITUACIÓN DE LOS DERECHOS HUMANOS EN LÍBANO, PRODUCIDA POR LAS OPERACIONES MILITARES ISRAELÍES". 11 DE AGOSTO DE 2006}

México desea expresar su preocupación por el deterioro de la situación en Medio Oriente a partir de la escalada de violencia derivada de los incidentes del 12 de julio de 2006, y de manera especial, por el sufrimiento de las víctimas inocentes, en particular de las mujeres y los niños.

México hace un llamado a incrementar los esfuerzos diplomáticos para llegar al cese de todas las hostilidades. Al mismo tiempo, le recuerda a todos los actores del conflicto que deben respetar en forma escrupulosa las obligaciones que derivan tanto del derecho internacional como del derecho internacional humanitario. El cese de las hostilidades, permitirá abrir un espacio de diálogo político para alcanzar una paz duradera.

Señor presidente,

México ha promovido la protección y respeto de todos los derechos humanos de todas las personas en todos los países. Asimismo, ha defendido que los derechos humanos deben garantizarse en todo tiempo, lugar y circunstancia.

También hemos reconocido que los derechos humanos no pueden suspenderse en tiempo de emergencia, de conflictos armados o en la lucha contra el terrorismo. Los derechos humanos sólo pueden limitarse en aquellos casos excepcionales en los que el propio derecho internacional de los derechos humanos y el derecho internacional humanitario lo permiten. En ese sentido, para México, existen derechos humanos que no pueden ser derogados por razones de necesidad militar.

Señor presidente,

El gobierno de México ha votado positivamente esta resolución motivado, principalmente, por la gravísima y penosa situación por la que atraviesa la población civil en la zona de conflicto. La resolución que se acaba de votar hace hincapié en hechos que si bien son reprobables, no comprenden la totalidad de los hechos de violencia que ocurren en la zona de conflicto. Desde el punto de vista de México, hubiera sido deseable que la resolución también incluyera una condena a los actos de violencia cometidos por las milicias de Hezbollah y a la necesidad de detener los ataques a la población civil israelí. Notamos con satisfacción que los copatrocinadores hayan incluido un llamado a todas las partes a respetar el derecho internacional humanitario. 
Además, mi delegación hubiera preferido que la resolución estableciera un mandato más amplio para la comisión de investigación de alto nivel, acorde con el objetivo del consejo de atender situaciones que afecten los derechos humanos en todos los países y no de manera selectiva o parcial.

Muchas gracias señor presidente.

\section{RESOLUCIÓN DEL CONSEJO DE SEGURIDAD. NACIONES UNIDAS. S/RES/1701 (2006). CONSEJO DE SEGURIDAD RESOLUCIÓN 1701 (2006)}

Aprobada por el Consejo de Seguridad en su 5511a. sesión, celebrada el 11 de agosto de 2006

El Consejo de Seguridad,

Recordando todas sus resoluciones anteriores sobre el Líbano, en particular las resoluciones 425 (1978), 426 (1978), 520 (1982), 1559 (2004), 1655 (2006), 1680 (2006) y 1697 (2006), así como las declaraciones de su Presidencia sobre la situación en el Líbano, en particular las declaraciones de 18 de junio de 2000 (S/PRST/2000/21), de 19 de octubre de 2004 (S/PRST/2004/36), de 4 de mayo de 2005 (S/PRST/2005/17), de 23 de enero de 2006 (S/PRST/2006/3) y de 30 de julio de 2006 (S/PRST/2006/35),

Expresando suma preocupación por la constante intensificación de las hostilidades en Líbano y en Israel desde el ataque lanzado por Hezbollah contra Israel el 12 de julio de 2006, que ya ha causado centenares de muertos y heridos en ambas partes, grandes daños en la infraestructura civil y centenares de millares de desplazados internos,

Haciendo hincapié en la necesidad de que se ponga fin a la violencia, pero al mismo tiempo haciendo hincapié en la necesidad de abordar con urgencia las causas que han dado origen a la crisis actual, entre otras cosas mediante la liberación sin condiciones de los soldados israelíes secuestrados,

Consciente de lo delicado de la cuestión de los prisioneros y alentando la labor destinada a solucionar urgentemente la cuestión de los prisioneros libaneses detenidos en Israel,

Acogiendo con satisfacción los esfuerzos desplegados por el primer ministro del Líbano y el compromiso del gobierno del Líbano, en su plan de siete puntos, de ampliar su autoridad sobre su territorio, a través de sus propias fuerzas armadas legítimas, de modo que no haya armas sin el consentimiento del gobierno del Líbano ni otra autoridad que no sea la del gobierno del Líbano, acogiendo con satisfacción también su compromiso con una fuerza de las Naciones Unidas 
aumentada y mejorada en términos de efectivos, equipo, mandato y alcance de sus operaciones, y teniendo presente la solicitud formulada en este plan de que las fuerzas israelies se retiren de inmediato del Libano meridional,

Decidido a tomar medidas para que esta retirada se produzca lo antes posible,

Tomando debida nota de las propuestas formuladas en el plan de siete puntos con respecto a la zona de las granjas de Shebaa,

Acogiendo con agrado la decisión unánime adoptada por el gobierno del Líbano el 7 de agosto de 2006 de desplegar una fuerza armada libanesa de 15.000 efectivos en el Líbano meridional a medida que el ejército israelí se repliega detrás de la Línea Azul y de pedir la asistencia de fuerzas adicionales de la Fuerza Provisional de las Naciones Unidas en el Líbano (FPNUL) según sea necesario, para facilitar la entrada de las fuerzas armadas libanesas en la región y de reiterar su intención de fortalecer las fuerzas armadas libanesas con el material que sea necesario para permitirle cumplir sus funciones,

Consciente de sus responsabilidades en lo que respecta a ayudar a establecer una cesación del fuego permanente y una solución a largo plazo del conflicto,

Determinando que la situación en el Líbano constituye una amenaza para la paz y la seguridad internacionales,

1. Pide una cesación total de las hostilidades basada, en particular, en la cesación inmediata por Hezbollah de todos los ataques y en la cesación inmediata por Israel de todas las operaciones militares ofensivas;

2. Después de la cesación total de las hostilidades, exhorta al gobierno del Líbano y a la FPNUL a que, conforme a lo autorizado en el párrafo 11, desplieguen en conjunto sus fuerzas en toda la región meridional y exhorta al gobierno de Israel a que, una vez iniciado ese despliegue, retire todas sus fuerzas del Líbano meridional en forma paralela;

3. Hace hincapié en la importancia de que se amplíe el control del gobierno del Líbano a todo el territorio libanés, de conformidad con las disposiciones de las resoluciones 1559 (2004) y 1680 (2006) y con las disposiciones pertinentes de los Acuerdos de Taif, para que ejerza su plena soberanía, de manera que no haya armas sin el consentimiento del gobierno del Líbano ni otra autoridad que la del gobierno del Líbano;

4. Reitera su firme apoyo al pleno respeto de la Línea Azul;

5. Reitera también su firme apoyo, como se recordó en todas sus resoluciones anteriores pertinentes, a la integridad territorial, la soberanía y la independencia política del Líbano dentro de sus fronteras reconocidas internacionalmente, según lo previsto en el Acuerdo General de Armisticio entre Israel y el Líbano de 23 de marzo de 1949; 
6. Exhorta a la comunidad internacional a que tome medidas inmediatas para prestar asistencia financiera y humanitaria al pueblo del Líbano, entre otras cosas, facilitando el regreso en condiciones de seguridad de las personas desplazadas y, bajo la autoridad del gobierno del Líbano, reabriendo los aeropuertos y puertos de conformidad con los párrafos 14 y 15, y la exhorta también a que considere la posibilidad de prestar más asistencia en el futuro para contribuir a la reconstrucción y el desarrollo del Líbano;

7. Afirma que todas las partes son responsables de velar por que no se adopte ninguna medida contraria al párrafo 1 que pueda afectar negativamente la búsqueda de una solución a largo plazo, el acceso humanitario a la población civil, incluido el tránsito sin peligro de los convoyes humanitarios, o el regreso voluntario y en condiciones de seguridad de las personas desplazadas, y exhorta a todas las partes a que cumplan con esa responsabilidad y cooperen con el Consejo de Seguridad;

8. Insta a Israel y al Líbano a que apoyen una cesación del fuego permanente y una solución a largo plazo basada en los siguientes principios y elementos:

- El pleno respeto de la Línea Azul por ambas partes;

- Arreglos de seguridad para impedir la reanudación de las hostilidades, en particular el establecimiento entre la Línea Azul y el río Litani de una zona libre de todo personal armado, bienes y armas, excepto los del gobierno del Líbano y de la FPNUL de acuerdo con lo autorizado en el párrafo 11, desplegados en esa zona;

- La plena aplicación de las disposiciones pertinentes de los Acuerdos de Taif y de las resoluciones 1559 (2004) y 1680 (2006) en que se exige el desarme de todos los grupos armados del Libano para que, de conformidad con la decisión del gobierno del Líbano de fecha 27 de julio de 2006, no haya más armas ni autoridad en el Líbano que las del Estado libanés;

- Ninguna fuerza extranjera presente en el Líbano sin el consentimiento de su gobierno;

- Ninguna venta ni suministro de armas y material conexo al Líbano, salvo con la autorización de su gobierno;

- La entrega a las Naciones Unidas de los mapas de minas terrestres en el Líbano que Israel todavía tenga en su poder;

9. Invita al secretario general a que apoye los esfuerzos para lograr lo antes posible acuerdos en principio del gobierno del Líbano y del gobierno de Israel 
sobre los principios y elementos para una solución a largo plazo descritos en el párrafo 8 , y expresa su intención de participar activamente;

10. Pide al secretario general que, en coordinación con los principales agentes internacionales y las partes interesadas, formule propuestas para aplicar las disposiciones pertinentes de los Acuerdos de Taif y de las resoluciones 1559 (2004) y 1680 (2006), incluido el desarme, y para el trazado de las fronteras internacionales del Líbano, especialmente en las zonas en las que la frontera es controvertida o incierta, ocupándose también de las granjas de Shebaa, y que le presente esas propuestas en un plazo de treinta días;

11. Decide, con el fin de aumentar y mejorar la fuerza en términos de efectivos, equipo, mandato y alcance de las operaciones, autorizar el aumento de los efectivos de la FPNUL a un máximo de 15.000 soldados, y que la fuerza, además de llevar a cabo su mandato de conformidad con sus resoluciones 425 (1978) y 426 (1978):

a) Vigile la cesación de las hostilidades;

b) Acompañe y apoye a las fuerzas armadas libanesas a medida que se despliegan en todo el sur, incluso a lo largo de la Línea Azul, mientras Israel retira a sus fuerzas armadas del Líbano de acuerdo con lo dispuesto en el párrafo 2;

c) Coordine sus actividades relacionadas con el apartado b) del párrafo 11 con el gobierno del Líbano y el gobierno de Israel;

d) Amplíe su asistencia para ayudar a asegurar el acceso humanitario a la población civil y el regreso voluntario y en condiciones de seguridad de las personas desplazadas;

e) Preste asistencia a las fuerzas armadas libanesas en la adopción de medidas para el establecimiento de la zona a que se hace referencia en el párrafo 8;

f) Preste asistencia al gobierno del Líbano, a solicitud de éste, en la aplicación del párrafo 14;

12. Actuando en apoyo de una solicitud del gobierno del Líbano de que se despliegue una fuerza internacional para ayudarlo a ejercer su autoridad en todo el territorio, autoriza a la FPNUL a que tome todas las medidas necesarias y que estime que están dentro de sus capacidades en las zonas de despliegue de sus fuerzas, para asegurarse de que su zona de operaciones no será utilizada para llevar a cabo actividades hostiles de ningún tipo, a que resista los intentos de impedirle por medios coercitivos cumplir las funciones que le incumben de conformidad con el mandato del Consejo de Seguridad, y a que proteja al personal, los servicios, las instalaciones y el equipo de las Naciones Unidas, vele por la seguridad y la libertad de circulación del personal de las Naciones Unidas y los trabajadores humanitarios y que, sin perjuicio de la responsabilidad del gobierno 
del Líbano, proteja a los civiles que se encuentren bajo amenaza inminente de sufrir violencia fisica;

13. Pide al secretario general que establezca con urgencia medidas para asegurar que la FPNUL pueda llevar a cabo las funciones previstas en la presente resolución, insta a los Estados miembros a que consideren la posibilidad de hacer contribuciones apropiadas a la FPNUL y a que respondan positivamente a las solicitudes de asistencia de la fuerza, y expresa su profundo reconocimiento a aquellos que han contribuido a la FPNUL en el pasado;

14. Exhorta al gobierno del Líbano a que asegure sus fronteras y otros puntos de ingreso para impedir la entrada en el Líbano de armas o material conexo sin su consentimiento y pide a la FPNUL que, de acuerdo con lo autorizado en el párrafo 11, preste asistencia al gobierno del Líbano cuando éste la solicite;

15. Decide además que todos los Estados adopten las medidas necesarias para impedir que sus nacionales o desde sus territorios o usando buques o aviones que enarbolen su pabellón:

a) Se vendan o suministren a cualquier entidad o persona del Líbano armas y material conexo de todo tipo, incluso armas y municiones, vehículos y equipo militares, equipo paramilitar, y piezas de repuesto para éstos, sea que tengan o no su origen en sus territorios; y

b) Se suministre a cualquier entidad o persona del Líbano asistencia o capacitación técnica relacionada con el suministro, la fabricación, el mantenimiento o el uso de los artículos enumerados en el apartado a) anterior; pero esas prohibiciones no serán aplicables a las armas y el material conexo, la capacitación o la asistencia autorizadas por el gobierno del Líbano o por la FPNUL según lo previsto en el párrafo 11;

16. Decide prorrogar el mandato de la FPNUL hasta el 31 de agosto de 2007, y expresa su intención de considerar en una resolución posterior nuevas mejoras del mandato y otras medidas para contribuir a la aplicación de una cesación del fuego permanente y una solución a largo plazo;

17. Pide al secretario general que informe al consejo en el plazo de una semana sobre la aplicación de esta resolución y posteriormente en forma periódica;

18. Recalca la importancia y la necesidad de lograr una paz amplia, justa y duradera en el Oriente Medio, basada en todas sus resoluciones pertinentes, entre ellas sus resoluciones 242 (1967), de 22 de noviembre de 1967, 338 (1973), de 22 de octubre de 1973, y 1515 (2003), de 19 de noviembre de 2003;

19. Decide seguir ocupándose activamente de la cuestión. 